\title{
The efficacy and safety of nivolumab in the treatment of advanced melanoma: a meta-analysis of clinical trials
}

This article was published in the following Dove Press journal:

OncoTargets and Therapy

16 March 2016

Number of times this article has been viewed

\section{Conghui Jin \\ Xunlei Zhang \\ Kuiling Zhao \\ Jun $X u$ \\ Min Zhao \\ Xiaohong Xu}

Department of Medical Oncology, Nantong Tumor Hospital, Nantong, People's Republic of China
Correspondence: Xiaohong Xu Nantong Tumor Hospital, 30 Tongyang North Road, Pingchao, Tongzhou District, Nantong, Jiangsu, People's Republic of China Email xhx107@hotmail.com
Background: Nivolumab has become a therapeutic regimen for the treatment of patients with advanced melanoma. The goal of this study was to assess the efficacy and safety of nivolumab in patients with advanced melanoma.

Methods: A systematic search from January 2008 to August 2015 with "nivolumab" and "advanced melanoma" as search terms was performed for possible clinical trials. According to the hazard ratio and the $95 \%$ confidence interval (CI) for progression-free survival (PFS), rates of objective response, complete response, partial response, rates of toxic effects, and the efficacy and safety of nivolumab were assessed. Using the software Review Manager (version 5.3) a meta-analysis was performed.

Results: There were four trials with 1,910 patients included. Based on the four trials, the pooled hazard ratio of PFS was $0.53(95 \% \mathrm{CI}, 0.43-0.66 ; P<0.001)$. The pooled risk ratio for the objective response rate, complete response, and partial response was $2.98 \%$ (95\% CI, $2.38 \%-3.73 \% ; P<0.001), 3.71 \%$ (95\% CI, 2.67\%-5.14\%; $P<0.001$ ), and 2.51\% (95\% CI, 2.12\%-2.99\%; $P<0.001)$, respectively. Nivolumab plus ipilimumab therapy significantly increased the risk of grade $3 / 4$ rash and fatigue.

Conclusion: Nivolumab-based therapy prolonged PFS in treatment of advanced melanoma, with less adverse effects. Nivolumab appears to be a favorable treatment option as a novel, targeted anticancer agent, for patients with advanced melanoma.

Keywords: nivolumab, advanced melanoma, meta-analysis

\section{Background}

In 2014, an estimated 76,100 patients were diagnosed with, and approximately 9,710 patients died of melanoma in the United States. ${ }^{1}$ Globally, the incidence of melanoma is estimated to be increasing by $3 \%-7 \%$ annually. ${ }^{2-5}$ However, these figures may represent a substantial underestimate, because many in situ cases and superficial melanomas treated in outpatient settings may go unreported. Many different treatment regimens are available for patients with stage III in-transit metastases. According to National Comprehensive Cancer Network guidelines for melanoma, for patients with stage III melanoma, treatment regimen options include: clinical trial, observation, or IFN- $\alpha .{ }^{1}$ But there is no consensus on the optimal approach. If available, enrollment in a clinical trial may be the preferred choice. The therapeutic treatment options for advanced melanoma are rapidly changing with the recent development of novel agents that have demonstrated better efficacy than traditional chemotherapy. ${ }^{6,7}$

During the past decade, the oncogenic drivers of cancer, the inhibitory and stimulatory pathways involved in immune responses, and evasion of immune surveillance by tumor cells have become better understood. Therefore, more targeted therapies are now 
under development. ${ }^{8-12}$ Novel therapeutic strategies, including targeted immunotherapies, represent major advances in the systemic treatment of cancers, such as for advanced melanoma. It has been shown that molecularly targeted therapies are efficacious for the treatment of certain cancers, such as for typically advanced melanoma and non-small-cell lung carcinoma. These molecularly targeted therapies include proto-oncogene BRAF and MEK inhibitors, antibodies that inhibit CTLA-4, and PD-1. Both BRAF and MEK inhibitors show a good response rate, but the median response duration is less than 1 year. ${ }^{13-17}$ It has been shown that ipilimumab, which inhibits CTLA-4, improves the survival rate to 2 years, compared with a vaccine control, among patients with advanced melanoma, whether previously treated or not. ${ }^{18,19}$ However, ipilimumab does have the potential for serious autoimmune toxicity. ${ }^{20}$

Nivolumab is a human $\mathrm{IgG}_{4}$ PD-1 immune checkpoint inhibitor antibody that disrupts T-cell activation and proliferation. ${ }^{21}$ Melanomas express high levels of PD-1. PD-1 binds with PD-L1(B7-H1) and PD-L2(B7-DC), expresses on antigen-presenting cells and human cancers, and delivers a negative signal to lymphocytes. ${ }^{22}$ B7-H1 expression confers immune resistance and interrupts PD-1 in murine tumor models. It is also highly upregulated in human tumors, where it inhibits local anti-tumor T-cell responses. ${ }^{23}$ Therefore, B7-H1 blockade is a strategy for cancer immunotherapy. There have been many trials evaluating the efficacy of treatment of advanced melanoma with nivolumab. ${ }^{24-27}$ Therefore, this study systematically combined data from published clinical trials to assess the efficacy and safety of nivolumab in the treatment of advanced melanoma, using the Preferred Reporting Items for Systematic Reviews and Meta-Analyses guidelines. ${ }^{28}$

\section{Materials and methods Data collection}

The aim of this meta-analysis was to assess the efficacy and safety of nivolumab in the treatment of advanced melanoma. The data were searched to identify clinical trials from Embase, PubMed (MEDLINE), and Cochrane Library, from January 2008 to August 2015 with "nivolumab" and "advanced melanoma" as search terms. Data were searched only in full texts which were available as original publications, and no meeting abstracts were selected. If necessary, we contacted the corresponding authors for further information.

\section{Study selection}

In this analysis, trials which met the following criteria were chosen: 1) articles with randomized controlled trials (RCTs) investigating the efficacy of nivolumab in the treatment of advanced melanoma; 2) RCTs having available data. Additionally, the nivolumab or nivolumab-based (nivolumab plus ipilimumab) therapy should be contained in the research group; 3 ) the data of progression-free survival (PFS) or overall survival (OS), objective response rate (ORR), complete response (CR), partial response (PR), stable disease, and adverse effects of nivolumab were reported; 4) there was at least one of the two endpoints (PFS, OS) reported; and, 5) the full text was available.

\section{Quality assessment}

The quality of selected clinical trials was evaluated according to the criteria presented in the Cochrane Handbookfor Systematic Reviews of Interventions (version 5.1; chapter 8), ${ }^{29}$ and appraised sequence generation, personnel and outcome assessors, allocation concealment, blinding of participants, addressing incomplete outcome data, and ensuring the absence of selective reporting and other sources of bias were included.

\section{Data extraction and presentation}

Study identifications (the first author and year of publication), patient details (age, sex, treatment comparison, and drug delivery mode), the number of patients in each trial, hazard ratio (HR) and their 95\% confidence intervals (CIs) for PFS or OS, rates of objective response, and adverse events (AEs) were included. We contacted the corresponding authors for additional data if HRs were not directly reported, or if that was not possible, we extracted additional data from survival curves.

\section{Statistical analysis}

The PFS and OS were considered to be primary outcomes of this meta-analysis. Pooled HRs were calculated for PFS and OS. The risk ratio (RR) was used as the effect size of the ORR, $\mathrm{CR}, \mathrm{PR}$ and AEs. Using the Q statistic to assess the presence of statistical heterogeneity, the $I^{2}$ statistic was assessed for the magnitude of heterogeneity. Fixed-effect models were used in all analyses unless heterogeneity existed $(P<0.1$ or $I^{2}>50 \%$ ). Data were considered to be significant if $P$-values were less than 0.05 , and all reported $P$-values were two-sided. The Cochrane Collaboration's Software Review Manager (version 5.3) was used to perform all statistical calculations.

\section{Results}

\section{Patient characteristics}

A total of 83 studies were searched from the published reports, and 51 were excluded after reviewing each publication. 
For the remaining 32 which met our criteria, including RCTs, single-arm studies, study protocols, or conference abstracts, 26 reports were excluded because they were abstracts with unavailable data or were ongoing studies. Finally, in the present meta-analysis, there were a total of 1,910 patients included in four RCTs ${ }^{24-27}$ selected. Figure 1 shows the article selection process, and the characteristics of the four articles selected and quality assessment of RCTs are summarized in Tables 1 and 2 .

\section{Efficacy}

\section{PFS}

As nivolumab is a newly approved drug, only one report presented OS in the four RCTs, thus, the efficacy of nivolumab was investigated with PFS and ORR in the treatment of melanoma.

All studies provided data on PFS. The combined HR for PFS was 0.55 (95\% CI, 0.50-0.61; $P<0.001)$. But there was mid-range heterogeneity among the trials $\left(P=0.01 ; I^{2}=69 \%\right)$. Therefore, a randomized effects model was undertaken. The results indicated there were significant differences in HRs $(\mathrm{HR}=0.53,95 \% \mathrm{CI}, 0.43-0.66 ; P<0.001 ; P$-value of heterogeneity $=0.01, I^{2}=69 \%$, Figure $2 \mathrm{~A}$ ), which indicated a $47 \%$ reduction in the risk of disease progression in patients treated with nivolumab or a nivolumab-based method.
The studies were divided into different therapeutic regimens, including the nivolumab plus ipilimumab trials, and the nivolumab trials, to identify the efficacy of nivolumab treatment in specific therapeutic regimens. For the total population, nivolumab was an effective treatment for melanoma The efficacy of nivolumab was tested in each subgroup (nivolumab plus ipilimumab subgroup and the nivolumab subgroup); and nivolumab was still found to be an effective treatment in both subgroups (Figure 2B).

\section{ORR}

All trials presented the information on ORR, and three studies provided the data for CR and PR. CR was defined as disappearance of all target lesions, and PR was defined as at least a $30 \%$ decrease in the sum of the diameter of the target lesions, taking as a reference the baseline sum diameter, according to the Response Evaluation Criteria in Solid Tumors. ${ }^{30}$ The aggregate RR for ORR (Figure 3A), CR (Figure 3B), and PR (Figure 3C) was 2.98\% (95\% CI, 2.38\%-3.73\%; $P<0.001)$, $3.71 \%(95 \%$ CI, $2.67 \%-5.14 \% ; P<0.001)$, and $2.51 \%(95 \%$ CI, $2.12 \%-2.99 \% ; P<0.001)$, respectively.

\section{AEs}

All studies provided data on multiple AEs after nivolumab treatment. Regarding grade 3/4 and all grade AEs, all four

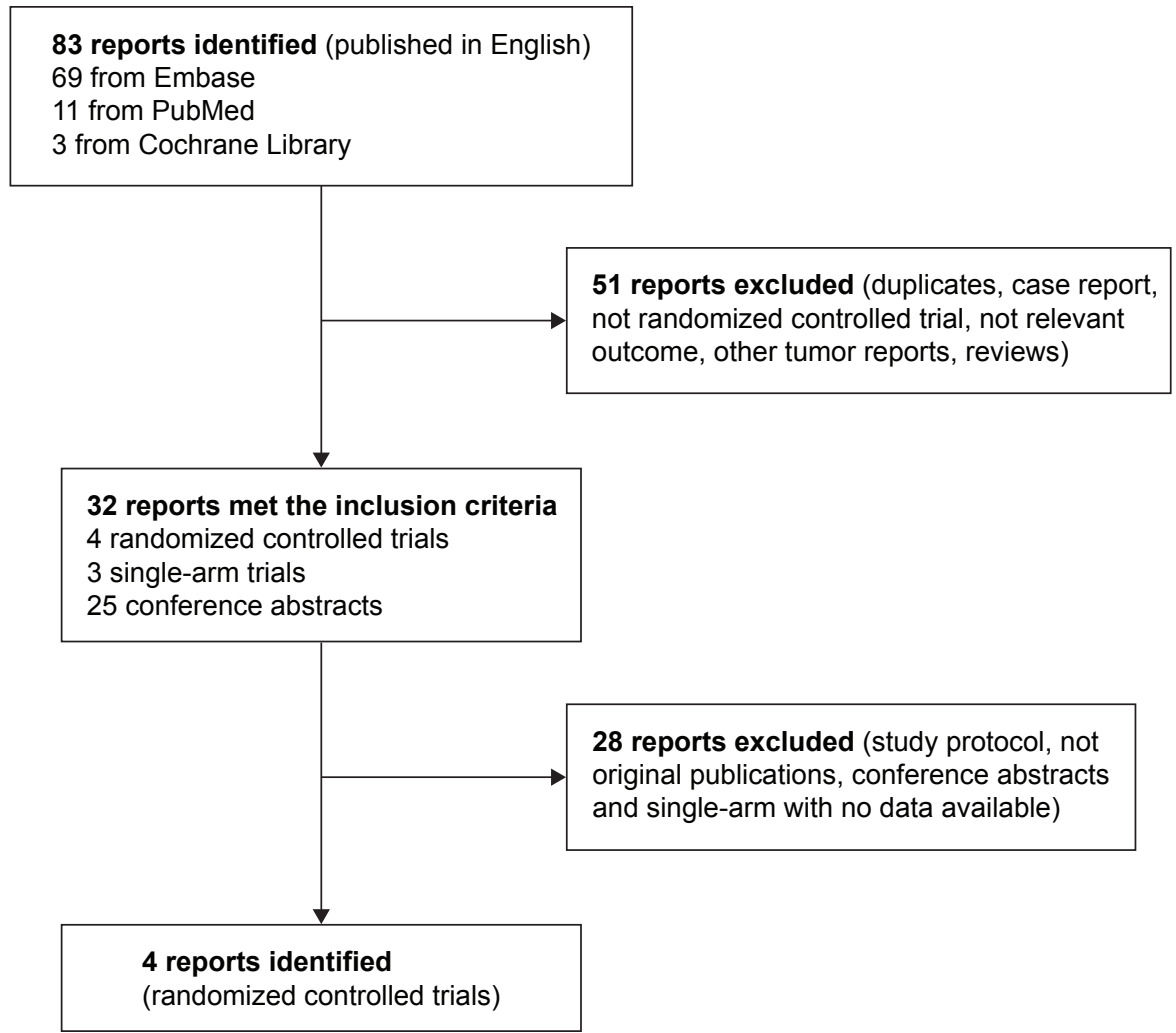

Figure I Chart of study identification, rejection, and selection in the meta-analysis. 
Table I The patients' characteristics of four clinical trials included

\begin{tabular}{|c|c|c|c|c|c|c|c|}
\hline Reference (year) & Group & $\begin{array}{l}\text { Patients } \\
\text { (N) }\end{array}$ & $\begin{array}{l}\text { Median age } \\
\text { (years) }\end{array}$ & $\begin{array}{l}\text { Sex } \\
(F ; n)\end{array}$ & $\begin{array}{l}\text { Metastasis } \\
\text { stage M Ic (n) }\end{array}$ & $\begin{array}{l}\text { LDH }>\text { ULN } \\
\text { (n) }\end{array}$ & $\begin{array}{l}\text { History of brain } \\
\text { metastases (n) }\end{array}$ \\
\hline \multirow[t]{6}{*}{ Postow et $\mathrm{a}^{25}(2015)$} & BRAF wild-type & & & & & & \\
\hline & Nivolumab + ipilimumab & 72 & 66 & 24 & 34 & 15 & 4 \\
\hline & Ipilimumab & 37 & 69 & 14 & 16 & 7 & 0 \\
\hline & All & & & & & & \\
\hline & Nivolumab + ipilimumab & 95 & 64 & 32 & 44 & 24 & 4 \\
\hline & Ipilimumab & 47 & 67 & 15 & 21 & 11 & 0 \\
\hline \multirow[t]{3}{*}{ Larkin et $\mathrm{al}^{24}(2015)$} & Nivolumab & 316 & 59 & 114 & 184 & 112 & 8 \\
\hline & Nivolumab + ipilimumab & 314 & 59 & 108 & 181 & 114 & II \\
\hline & Ipilimumab & 315 & 61 & 113 & 183 & 115 & 15 \\
\hline \multirow[t]{2}{*}{ Robert et $\mathrm{al}^{26}(2015)$} & Nivolumab & 210 & 64 & 89 & 128 & 79 & 7 \\
\hline & Dacarbazine & 208 & 66 & 83 & 127 & 74 & 8 \\
\hline \multirow[t]{2}{*}{ Weber et $\mathrm{al}^{27}(2015)$} & Nivolumab & 272 & 59 & 96 & 202 & 139 & 53 \\
\hline & Dacarbazine \pm paclitaxel & 133 & 62 & 48 & 102 & 46 & 18 \\
\hline
\end{tabular}

Abbreviations: F, female; LDH, lactate dehydrogenase; ULN, upper limits of normal.

studies reported data of fatigue, diarrhea, pruritus, nausea, and vomiting. Data of rashes were not available in one study. There was serious heterogeneity in analysis of four trials; therefore we divided these into two subgroups, the nivolumab plus ipilimumab subgroup versus the ipilimumab, ${ }^{24,26}$ and nivolumab subgroup versus the dacarbazine or dacarbazinebased therapy subgroup..$^{25,27}$ Results of grade $3 / 4$ and all grade AEs are presented in Table 3.

\section{Discussion}

The efficacy and safety of nivolumab in the treatment of advanced melanoma were assessed in this meta-analysis. The aggregated HR of PFS and RR of ORR showed that nivolumab treatment had a generally prolonged PFS and improved response rate, which strongly indicated the effectiveness of nivolumab or nivolumab-based therapy regimens in patients with advanced melanoma.

The National Comprehensive Cancer Network guidelines for melanoma state that therapy for advanced melanoma is still under study. ${ }^{31}$ Targeted drugs for melanoma treatment, including ipilimumab, a preferred drug for advanced melanoma (category 1), have the potential for significant immune-mediated complications. Ipilimumab should be used with extreme caution due to its serious underlying autoimmune effects. However, evidence is limited and no consensus is available on the optimal approach for advanced melanoma treatment. Enrollment in a clinical trial, if available, is the preferred choice. Recently, the PD-1 inhibitor, nivolumab has gained attention, and Phase 3 trials were done to evaluate its efficacy and safety. However, because nivolumab is a newly developed drug, there has only been a single trial providing OS that showed extended survival.

In our study, two trials indicated that nivolumab plus ipilimumab versus ipilimumab, in advanced melanoma treatment, had aggregated HR of PFS, indicating the combined therapy was more effective than the single ipilimumab therapy. While the other two trials combined the nivolumab versus dacarbazine or dacarbazine-based therapy, the results were still significantly improved.

The presented trials analyzed grade $3 / 4$ side effects, and significant differences were found for fatigue. However, some differences were due to server heterogeneity, therefore we divided the results into two subgroups. In the combined nivolumab group we found significant differences in rash and fatigue in grade $3 / 4$, but no significant differences in the single-nivolumab group. In the single-nivolumab group, no significant differences in grade 3/4 were shown; however, pruritus, vomiting, and nausea had significant differences in all grades. More pruritus was noted in the single-nivolumab

Table 2 The quality assessment of four randomized controlled trials included

\begin{tabular}{|c|c|c|c|c|c|c|c|}
\hline Reference & $\begin{array}{l}\text { Patients } \\
\text { (N) }\end{array}$ & $\begin{array}{l}\text { Adequate sequence } \\
\text { generation }\end{array}$ & $\begin{array}{l}\text { Allocation } \\
\text { concealment }\end{array}$ & Blinding & $\begin{array}{l}\text { Incomplete outcome } \\
\text { data addressed }\end{array}$ & $\begin{array}{l}\text { Free of selective } \\
\text { reporting }\end{array}$ & $\begin{array}{l}\text { Free of } \\
\text { other bias* }\end{array}$ \\
\hline Postow et $\mathrm{al}^{25}$ & 142 & Yes & Yes & Yes & Yes & Yes & Yes \\
\hline Larkin et $\mathrm{al}^{24}$ & 945 & Yes & Yes & Yes & Yes & Yes & Yes \\
\hline Robert et $\mathrm{al}^{26}$ & 418 & Yes & Yes & Yes & Yes & Yes & Yes \\
\hline Weber et $\mathrm{al}^{27}$ & 405 & Yes & Yes & Yes & Yes & Yes & Yes \\
\hline
\end{tabular}

Note: *Other bias refers to selective bias and measurement bias. 


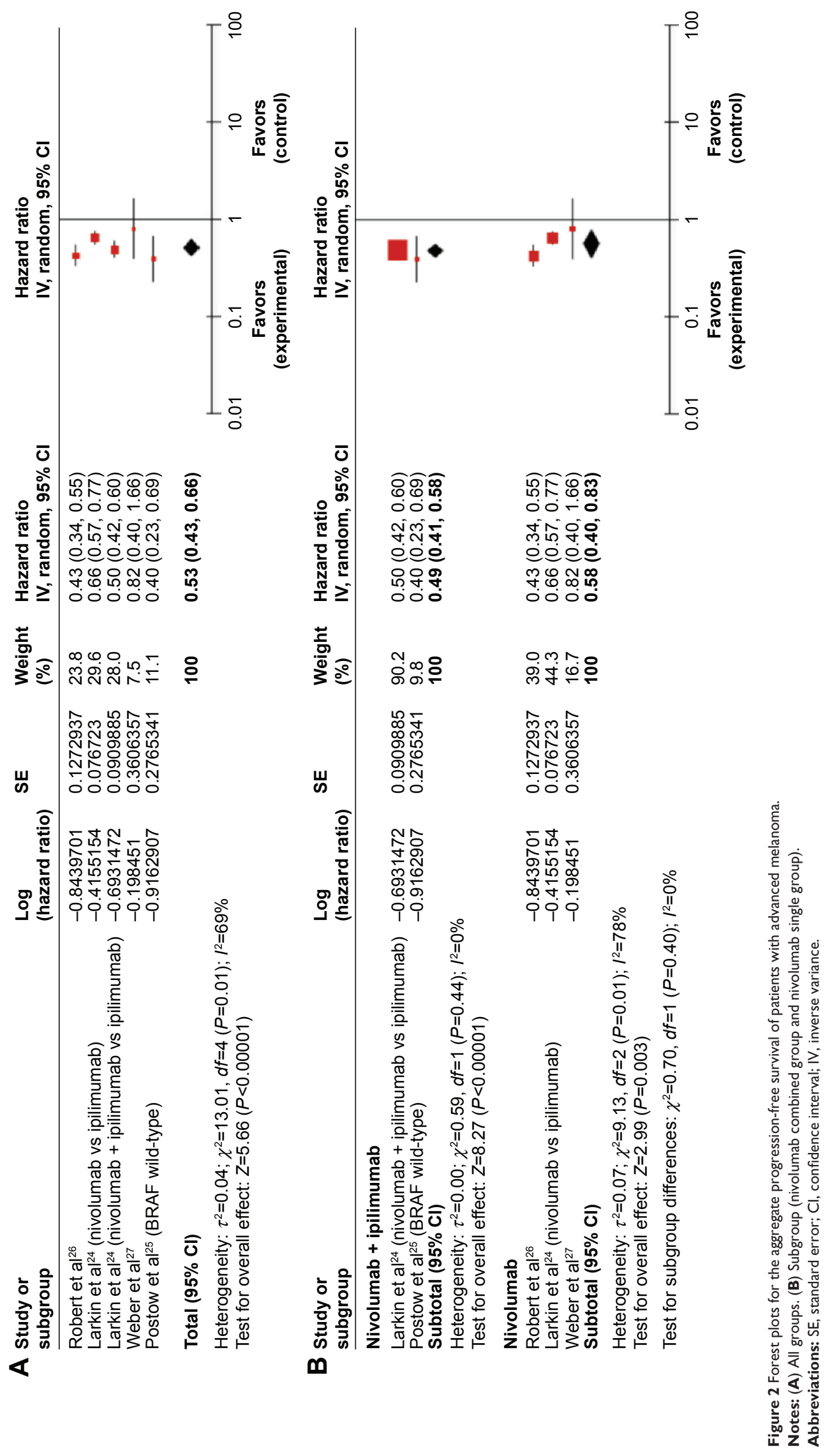




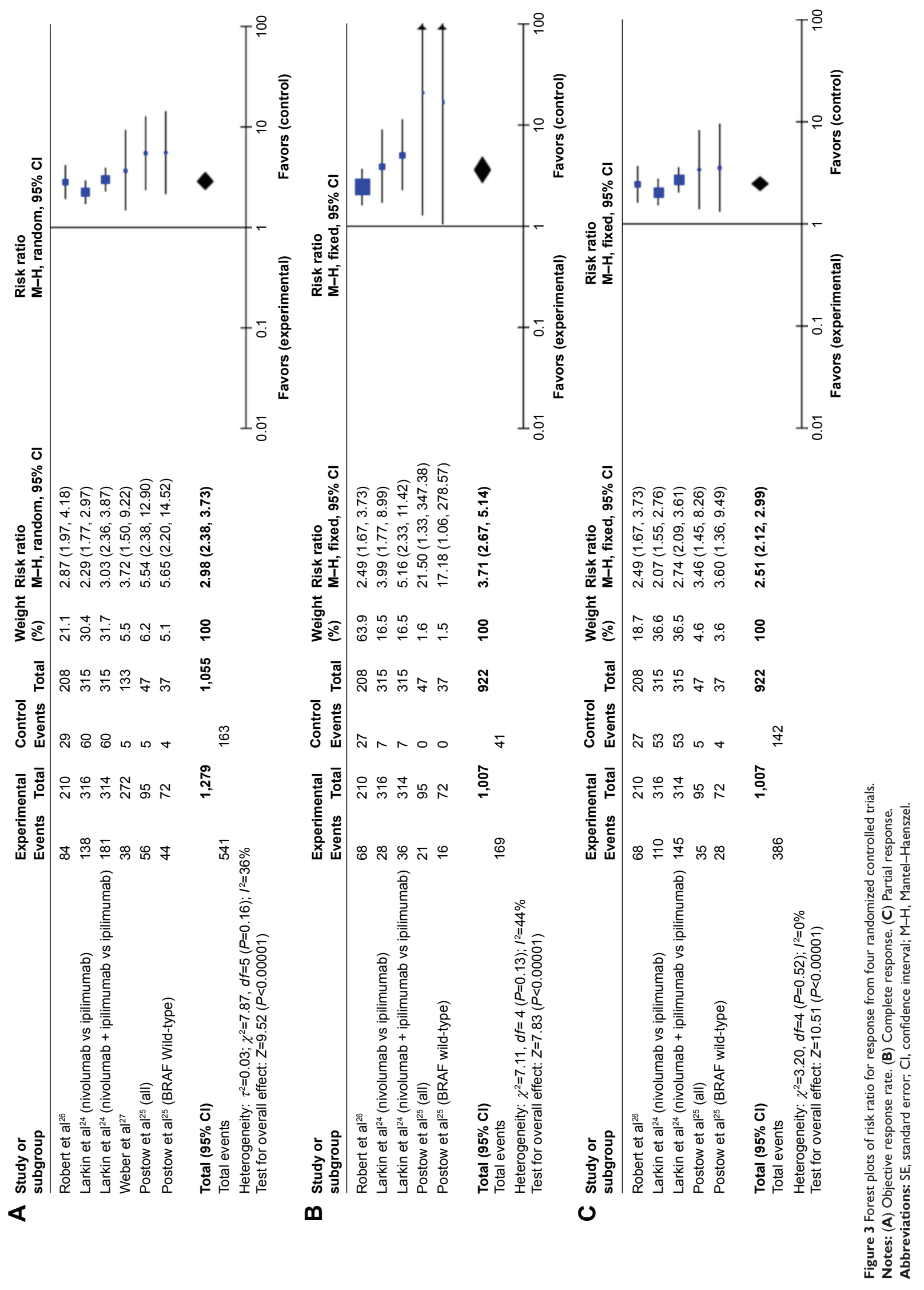


Table 3 The toxic effects of nivolumab in advanced melanoma

\begin{tabular}{|c|c|c|c|c|}
\hline \multirow[t]{2}{*}{ Adverse events } & \multicolumn{2}{|c|}{ Nivolumab + ipilimumab vs ipilimumab } & \multicolumn{2}{|c|}{ Nivolumab vs dacarbazine } \\
\hline & Pooled $\mathrm{RR}$ and $95 \% \mathrm{Cl}$ & $P$-value & Pooled RR and $95 \% \mathrm{Cl}$ & $P$-value \\
\hline \multicolumn{5}{|l|}{ Grade 3 and 4} \\
\hline Diarrhea & $1.38(0.85-2.26)$ & 0.19 & $0.70(0.09-5.4 \mathrm{I})$ & 0.73 \\
\hline Rash & $2.70(1.11-6.57)$ & 0.03 & NA* & NA* \\
\hline Pruritus & $4.27(0.75-24.35)$ & 0.10 & $2.99(0.12-73.71)$ & 0.50 \\
\hline Fatigue & $4.5 I(1.44-14.15)$ & 0.01 & $0.23(0.05-1.01)$ & 0.05 \\
\hline Vomiting & $5.41(1.03-28.34)$ & 0.05 & $0.45(0.08-2.46)$ & 0.36 \\
\hline Nausea & $2.30(0.65-8.15)$ & 0.20 & None $^{\wedge}$ & None $^{\wedge}$ \\
\hline \multicolumn{5}{|l|}{ All grades } \\
\hline Diarrhea & $1.32(1.10-1.59)$ & 0.003 & $0.68(0.3 \mid-1.50)$ & 0.35 \\
\hline Rash & $1.28(1.05-1.56)$ & 0.01 & NA* & $N A^{*}$ \\
\hline Pruritus & $0.99(0.8 I-1.21)$ & 0.93 & $4.96(1.47-16.72)$ & 0.01 \\
\hline Fatigue & $2.30(0.33-15.83)$ & 0.40 & $5.69(0.05-636.7)$ & 0.47 \\
\hline Vomiting & $1.91(1.25-2.92)$ & 0.003 & $0.27(0.17-0.43)$ & 0.000 \\
\hline
\end{tabular}

Notes: NA*: not provided; none^: there were no patients with nausea.

Abbreviations: $\mathrm{RR}$, risk ratio; $\mathrm{Cl}$, confidence interval.

group, but less vomiting and nausea was noted than in the dacarbazine or dacarbazine-based therapy group. Therefore, the reported side effects associated with nivolumab were mostly mild to moderate.

Whether combined nivolumab treatment is more efficacious than single nivolumab remains unknown. One trial $^{25}$ that divided treatments into three groups, the nivolumab plus ipilimumab, nivolumab, and ipilimumab therapy groups, showed that combined therapy prolonged the median PFS more than single nivolumab therapy, but the PD-1 positive group showed the same efficacy. However for adverse effects, the combined therapy presented more adverse effects such as rash and fatigue, than the single therapy. It has been reported that single ipilimumab treatment induced immune-related adverse events (irAEs), including dermatitis, hepatitis, colitis, and endocrinopathies, such as adrenalitis, thyroiditis, and hypophysitis. ${ }^{20,32-34}$ Treatment guidelines were reported to help physicians manage ipilimumab irAEs, which could have contributed to the decrease of grade 3/4 AEs and drug-related deaths. ${ }^{35}$ However, nivolumab plus ipilimumab therapy might increase the occurrence rate of irAEs. Further trials are necessary to determine whether the combined therapy is more efficacious than single therapy.

Because no aggregated HR for OS is available, we cannot evaluate an accurate estimate of time of OS. Additional subgroup population studies are therefore necessary to analyze the efficacy and safety of nivolumab.

\section{Conclusion}

In conclusion, nivolumab provides a statistically significant and clinically relevant extension of life in patients with advanced melanoma. Toxicity analyses suggest that nivolumab side effects are mostly mild to moderate. Further randomized, blinded, placebo-controlled trials are required to compare the efficacy and safety of nivolumab with other treatments used for advanced melanoma.

\section{Disclosure}

The authors report no conflicts of interest in this work.

\section{References}

1. National Comprehensive Cancer Network [homepage on the Internet]. NCCN Clinical Practice Guidelines in Oncology (NCCN Guidelines $\left.{ }^{\circledR}\right)$ : melanoma version 3.2015. NCCN; 2015. Available from: http://www. nccn.org/professionals/physician_gls/f_guidelines.asp\#site. Accessed February 3, 2016.

2. Johnston KM, McPherson E, Osenenko K, et al. Cost-effectiveness of therapies for melanoma. Expert Rev Pharmacoecon Outcomes Res. 2015;15(2):229-242.

3. Fox MC, Lao CD, Schwartz JL, et al. Management options for metastatic melanoma in the era of novel therapies: a primer for the practicing dermatologist: part II: management of stage IV disease. $J$ Am Acad Dermatol. 2013;68(1):13.e1-13.

4. World Health Organization [homepage on the Internet]. Ultraviolet radiation and the INTERSUN Programme. WHO; 2012. Available from: http://www.who.int/uv/en/. Accessed February 3, 2016.

5. Dummer R, Hauschild A, Guggenheim M, et al. Cutaneous melanoma: ESMO Clinical Practice Guidelines for diagnosis, treatment and follow-up. Ann Oncol. 2012;23 Suppl 7:vii86-vii91.

6. Niezgoda A, Niezgoda P, Czajkowski R. Novel Approaches to Treatment of Advanced Melanoma: A Review on Targeted Therapy and Immunotherapy. Biomed Res Int. 2015;2015:851387.

7. Saranga-Perry V, Ambe C, Zager JS, Kudchadkar RR. Recent developments in the medical and surgical treatment of melanoma. CA Cancer J Clin. 2014;64(3):171-185.

8. Weide B, Di Giacomo AM, Fonsatti E, Zitvogel L. Immunologic correlates in the course of treatment with immunomodulating antibodies. Semin Oncol. 2015;42(3):448-458.

9. Luke JJ, Ott PA. PD-1 pathway inhibitors: the next generation of immunotherapy for advanced melanoma. Oncotarget. 2015;6(6):3479-3492.

10. Antonia SJ, Larkin J, Ascierto PA. Immuno-oncology combinations: a review of clinical experience and future prospects. Clin Cancer Res. 2014;20(24):6258-6268. 
11. Shih K, Arkenau HT, Infante JR. Clinical impact of checkpoint inhibitors as novel cancer therapies. Drugs. 2014;74(17):1993-2013.

12. Tsai KK, Daud AI. The role of anti-PD-1/PD-L1 Agents in Melanoma: Progress to Date. Drugs. 2015;75(6):563-575.

13. McArthur GA, Chapman PB, Robert C, et al. Safety and efficacy of vemurafenib in BRAF V600E and BRAF V600K mutation-positive melanoma (BRIM-3): extended follow-up of a phase 3, randomised, open-label study. Lancet Oncol. 2014;15(3):323-332.

14. Flaherty KT, Robert C, Hersey P, et al. Improved survival with MEK inhibition in BRAF-mutated melanoma. N Engl J Med. 2012; 367(2):107-114.

15. Hauschild A, Grob JJ, Demidov LV, et al. Dabrafenib in BRAF-mutated metastatic melanoma: a multicentre, open-label, phase 3 randomised controlled trial. Lancet. 2012;380(9839):358-365.

16. Sosman JA, Kim KB, Schuchter L, et al. Survival in BRAF V600-mutant advanced melanoma treated with vemurafenib. $N$ Engl J Med. 2012; 366(8):707-714.

17. Chapman PB, Hauschild A, Robert C, et al. Improved survival with vemurafenib in melanoma with BRAF V600E mutation. $N$ Engl $J$ Med. 2011;364(26):2507-2516.

18. Hodi FS, O'Day SJ, McDermott DF, et al. Improved survival with ipilimumab in patients with metastatic melanoma. $N$ Engl J Med. 2010; 363(8):711-723.

19. Robert C, Thomas L, Bondarenko I, et al. Ipilimumab plus dacarbazine for previously untreated metastatic melanoma. $N$ Engl J Med. 2011; 364(26):2517-2526.

20. Wang $C$, Thudium KB, Han $M$, et al. In vitro characterization of the anti-PD-1 antibody nivolumab, BMS-936558, and in vivo toxicology in non-human primates. Cancer Immunol Res. 2014;2(9):846-856.

21. Haanen JB, Thienen Hv, Blank CU. Toxicity patterns with immunomodulating antibodies and their combinations. Semin Oncol. 2015; 42(3):423-428.

22. Topalian SL, Mario S, McDermott DF, et al. Survival, durable tumor remission, and long-term safety in patients with advanced melanoma receiving nivolumab. J Clin Oncol. 2014;32(10):1020-1030.

23. Topalian SL, Drake CG, Pardoll DM. Targeting the PD-1/B7-H1 (PD-L1) pathway to activate anti-tumor immunity. Curr Opin Immunol. 2012;24(2):207-212.

24. Larkin J, Chiarion-Sileni V, Gonzalez R, et al. Combined Nivolumab and Ipilimumab or Monotherapy in Untreated Melanoma. N Engl J Med. 2015;373(1):23-34.
25. Postow MA, Chesney J, Pavlick AC, et al. Nivolumab and ipilimumab versus ipilimumab in untreated melanoma. $N$ Engl J Med. 2015; 372(21):2006-2017.

26. Robert C, Long GV, Brady B, et al. Nivolumab in previously untreated melanoma without BRAF mutation. $N$ Engl J Med. 2015;372(4): 320-330.

27. Weber JS, D'Angelo SP, Minor D, et al. Nivolumab versus chemotherapy in patients with advanced melanoma who progressed after anti-CTLA-4 treatment (CheckMate 037): a randomised, controlled, open-label, phase 3 trial. Lancet Oncol. 2015;16(4):375-384.

28. Moher D, Cook DJ, Eastwood S, et al. Improving the quality of reports of meta-analyses of randomised controlled trials: the QUOROM statement. Lancet. 1999;354(9193):1896-1900.

29. Higgins JPT, Green S, editors. Cochrane handbook for systematic reviews of interventions version 5.1.0. The Cochrane Collaboration; 2011. Available from: http://www.cochrane-handbook.org. Accessed March 2, 2016.

30. Eisenhauer EA, Therasse P, Bogaerts J, et al. New response evaluation criteria in solid tumours: revised RECIST guideline (version 1.1). Eur J Cancer. 2009;45(2):228-247.

31. Thompson JA. Treatment options expanding for advanced melanoma. J Natl Compr Canc Netw. 2015;13(5 Suppl):673-675.

32. Delyon J, Maio M, Lebbé C. The ipilimumab lesson in melanoma: achieving long-term survival. Semin Oncol. 2015;42(3):387-401.

33. Delyon J, Mateus C, Lefeuvre D, et al. Experience in daily practice with ipilimumab for the treatment of patients with metastatic melanoma: an early increase in lymphocyte and eosinophil counts is associated with improved survival. Ann Oncol. 2013;24(6):1697-1703.

34. Ascierto PA, Simeone E, Sileni VC, et al. Clinical experience with ipilimumab $3 \mathrm{mg} / \mathrm{kg}$ : real-world efficacy and safety data from an expanded access programme cohort. $J$ Transl Med. 2014;12(1):116.

35. Weber JS, Dummer R, de Pril V, et al. Patterns of onset and resolution of immune-related adverse events of special interest with ipilimumab. Cancer. 2013;119(9):1675-1682.
OncoTargets and Therapy

\section{Publish your work in this journal}

OncoTargets and Therapy is an international, peer-reviewed, open access journal focusing on the pathological basis of all cancers, potential targets for therapy and treatment protocols employed to improve the management of cancer patients. The journal also focuses on the impact of management programs and new therapeutic agents and protocols on

\section{Dovepress}

patient perspectives such as quality of life, adherence and satisfaction The manuscript management system is completely online and includes a very quick and fair peer-review system, which is all easy to use. Visit http://www.dovepress.com/testimonials.php to read real quotes from published authors. 